International Journal of Pure and Applied Mathematics

Volume 108 No. 2 2016, 215-226

ISSN: 1311-8080 (printed version); ISSN: 1314-3395 (on-line version)

url: http://www.ijpam.eu

doi: 10.12732/ijpam.v108i2.3

\title{
OPTIMAL SYSTEM AND SYMMETRY REDUCTION OF THE $(1+1)$ DIMENSIONAL SAWADA-KOTERA EQUATION
}

\author{
N. Kadkhoda ${ }^{1}$, H. Jafari ${ }^{2,3}$, G.M. Moremedi ${ }^{3}$, D. Baleanu ${ }^{4,5}$ \\ ${ }^{1}$ Department of Mathematics \\ Faculty of Basic Sciences \\ Bozorgmehr University Of Qaenat \\ Qaenat, IRAN \\ ${ }^{2}$ Department of Mathematics \\ University of Mazandaran \\ Babolsar, IRAN \\ ${ }^{3}$ Department of Mathematical Sciences \\ University of South Africa \\ UNISA0003, Pretoria, SOUTH AFRICA \\ ${ }^{4}$ Department of Mathematics and Computer Sciences \\ Faculty of Art and Sciences \\ Cankaya University, Ankara, TURKEY \\ ${ }^{5}$ Institute of Space Sciences \\ 76900 Magurele-Bucharest, ROMANIA
}

\begin{abstract}
We study the nonlinear fifth order $(1+1)$ dimensional Sawada-Kotera equation using Lie symmetry group. For this equation Lie point symmetry operators and optimal system are obtained. We determine the corresponding invariant solutions and reduced equations using obtained infinitesimal generators.
\end{abstract}

AMS Subject Classification: 70G65

Key Words: Lie symmetry, optimal system, Sawada-Kotera equation, group-invariant solutions

Received: September 4, 2015

Published: June 18, 2016

$\S$ Correspondence author (c) 2016 Academic Publications, Ltd. url: www.acadpubl.eu 


\section{Introduction}

Nonlinear phenomena are very important in applied mathematics and physics. They appear in various scientific fields such as biology, signal processing, viscoelastic materials, fluid mechanics, optical fiber, and so on. Some years ago, researchers have provided many methods for obtaining the numerical and analytical solutions of nonlinear equations, such as tanh function method, extended tanh-function method $[1,2],\left(\frac{G^{\prime}}{G}\right)$-expansion method [3, 4], Sine-cosine method $[5,6]$, Simplest equation method[7] and so on. In this paper we study the Sawada-Kotera equation, namely

$$
u_{t}+5 u^{2} u_{x}+5 u_{x} u_{x x}+2 u u_{x x x}+u_{x x x x x}=0 .
$$

Sawada and Kotera proposed it more than thirty years ago [8]. Because many various properties are satisfied for this equation, much effort has been made about its exact solutions.

For example, Fuchssteiner and Oevel studied its biHamiltonian structure [9], and a Darboux transformation was obtained for this system [10, 11], Liu and Dai[12] accomplished the Hirota's bilinear method to obtain exact solutions of the same equation. Feng and Zheng [13] investigated this equation to establish traveling wave solutions via the $\left(\frac{G^{\prime}}{G}\right)$-expansion method. In [14], Wazwaz implemented the extended tanh method for constructing analytical solutions of the same equation.

The outline of the paper is as follows. In Section 2 we discuss the methodology of Lie symmetry analysis of the Sawada-Kotera equation. Then in Section 3 we describe the classical symmetries of the Sawada-Kotera equation and we obtain the Lie point symmetries of this equation. In Section 4 we explain the Group Invariant solutions. In Section 5 the optimal system are obtained of the one-dimensional subalgebras of the Sawada-Kotera equation. In Section 6 we obtain symmetry reduction and differential invariants for the Sawada-Kotera equation. Finally, concluding remarks are summarized in Section 7.

\section{Symmetry Group Analysis}

Nonlinear partial differential equations occur in most phenomenon in nature and finding exact solutions of these equations is very important. We know that some of these equations are very difficult to solve, but much effort has been made to construct the analytical solutions for them. Symmetry is one of the most important methods of differential equations to obtain the exact solutions. 
The invariance of the differential equation under infinitesimal operators is an essential concept of the Lie theory.

Sophus Lie, introduced Lie groups to solve differential equations in the nineteenth century, when he discovered that the differential equations are invariant under the continuous groups of transformations. The differential equations can be reduced to simpler equations using symmetries of them. In the last century, many researchers have developed applications of the Lie group method, some of the mathematicians who have studied in this field are Ibragimov [15], Ovsiannikov [16], Olver [17], Baumann [18] and Bluman [19].

Now let us consider a system of partial differential equations as follows[20]:

$$
\Lambda_{\nu}\left(x, u^{(n)}\right)=0, \quad \nu=1,2, \ldots, \ell,
$$

where $u=\left(u^{1}, u^{2}, \ldots, u^{q}\right), x=\left(x^{1}, x^{2}, \ldots, x^{p}\right), u^{(n)}$ denotes all the derivatives of $u$ of all orders from 0 to $n$.

The one-parameter Lie group of infinitesimal transformations of the system (2) is given by

$$
\begin{aligned}
& x^{* i}=x^{i}+\varepsilon \xi^{i}(x, u)+O\left(\varepsilon^{2}\right) ; \quad i=1,2, \ldots, p, \\
& u^{* \alpha}=u^{\alpha}+\varepsilon \eta^{\alpha}(x, u)+O\left(\varepsilon^{2}\right) ; \quad \alpha=1,2, \ldots, q,
\end{aligned}
$$

where $\varepsilon$ is the group parameter. The Lie algebra of (2) is spanned by vector field

$$
X=\sum_{i=1}^{p} \xi^{i}(x, u) \frac{\partial}{\partial x^{i}}+\sum_{\alpha=1}^{q} \eta^{\alpha}(x, u) \frac{\partial}{\partial u^{\alpha}} .
$$

The n-th order prolongation of $X$ is given by:

$$
X^{(n)}=\sum_{i=1}^{p} \xi^{i}(x, u) \frac{\partial}{\partial x^{i}}+\sum_{\alpha=1}^{q} \eta^{\alpha}(x, u) \frac{\partial}{\partial u^{\alpha}}+\sum_{\alpha=1}^{q} \sum_{J} \eta_{J}^{\alpha}\left(x, u^{(n)}\right) \frac{\partial}{\partial u_{J}^{\alpha}}
$$

Where $J=\left(i_{1}, \ldots, i_{k}\right), 1 \leq i_{k} \leq p, 1 \leq k \leq n$, and the sum is over all $J^{\prime} s$ of order $0<\# J \leq n$. If $\# J=k$, the coefficient $\eta_{J}^{\alpha}$ of $\frac{\partial}{\partial u_{J}^{\alpha}}$ will depend only on $k$-th and lower order derivatives of $u$, and

$$
\eta_{\alpha}^{J}\left(x, u^{(n)}\right)=D_{J}\left(\eta_{\alpha}-\sum_{i=1}^{p} \xi^{i} u_{i}^{\alpha}\right)+\sum_{i=1}^{p} \xi^{i} u_{J, i}^{\alpha}
$$

where $u_{i}^{\alpha}=\frac{\partial u^{\alpha}}{\partial x^{i}}$ and $u_{J, i}^{\alpha}=\frac{\partial u_{J}^{\alpha}}{\partial x^{2}}$. 


\section{Classical Symmetries of the Sawada-Kotera Equation}

The one-parameter Lie group of infinitesimal transformations of the SawadaKotera Equation in $x, t, u$ is given by

$$
\begin{aligned}
& x^{*}=x+\varepsilon \xi^{1}(x, t, u)+O\left(\varepsilon^{2}\right), \\
& t^{*}=t+\varepsilon \xi^{2}(x, t, u)+O\left(\varepsilon^{2}\right), \\
& u^{*}=u+\varepsilon \eta(x, t, u)+O\left(\varepsilon^{2}\right) .
\end{aligned}
$$

where $\varepsilon$ is the group parameter, and the Lie algebra of (1) is spanned by vector field of the form:

$$
X=\xi^{1}(x, t, u) \frac{\partial}{\partial x}+\xi^{2}(x, t, u) \frac{\partial}{\partial t}+\eta(x, t, u) \frac{\partial}{\partial u} .
$$

The fifth prolongation of $X$ is given by

$$
\begin{aligned}
X^{(5)}=X & +\eta^{x} \frac{\partial}{\partial u_{x}}+\eta^{t} \frac{\partial}{\partial u_{t}}+\eta^{2 x} \frac{\partial}{\partial u_{2 x}}+\eta^{x t} \frac{\partial}{\partial u_{x t}}+\eta^{2 t} \frac{\partial}{\partial u_{2 t}} \\
& +\eta^{3 x} \frac{\partial}{\partial u_{3 x}}+\ldots+\eta^{3 t} \frac{\partial}{\partial u_{3 t}}+\eta^{4 x} \frac{\partial}{\partial u_{4 x}}+\ldots+\eta^{4 t} \frac{\partial}{\partial u_{4 t}} \\
& +\eta^{5 x} \frac{\partial}{\partial u_{5 x}}+\ldots+\eta^{5 t} \frac{\partial}{\partial u_{5 t}}
\end{aligned}
$$

with coefficients

$$
\eta^{J}=D_{J}\left(\eta-\sum_{i=1}^{2} \xi^{i} u_{i}^{\alpha}\right)+\sum_{i=1}^{2} \xi^{i} u_{J, i}^{\alpha},
$$

where $J=\left(i_{1}, \ldots, i_{k}\right), 1 \leq i_{k} \leq 2,1 \leq k \leq 5$, and the sum is over all $J^{\prime} s$ of order $0<\# J \leq 5$. Using the fifth prolongation $\left(X^{(5)}\right)$ to Eq.(1)

$$
\left.X^{(5)}\left(u_{t}+5 u^{2} u_{x}+5 u_{x} u_{x x}+2 u u_{x x x}+u_{x x x x x}\right)\right|_{(1)=0}=0,
$$

we can obtain $\xi^{1}, \xi^{2}$ and $\eta$.

Theorem 1. The Lie algebra of Eq.(1)(Sawada-Kotera equation) is generated by the vector field

$$
X=\xi^{1}(x, t, u) \frac{\partial}{\partial x}+\xi^{2}(x, t, u) \frac{\partial}{\partial t}+\eta(x, t, u) \frac{\partial}{\partial u} .
$$

where

$$
\xi^{1}=\frac{1}{5} c_{1} x+c_{3}
$$




$$
\begin{aligned}
& \xi^{2}=c_{1} t+c_{2} \\
& \eta=-\frac{2}{5} c_{1} u
\end{aligned}
$$

and $c_{1}, c_{2}, c_{3}$ are arbitrary constants.

Proof. we Expand the Eq.(10) and then we solve the obtained set of linear differential equations using the Maple. this completes the proof.

Corollary 2. Lie point symmetries of the Sawada-Kotera equation are

$$
\begin{aligned}
& X_{1}=x \frac{\partial}{\partial x}+5 t \frac{\partial}{\partial t}-2 u \frac{\partial}{\partial u}, \\
& X_{2}=\frac{\partial}{\partial x} \\
& X_{3}=\frac{\partial}{\partial t} .
\end{aligned}
$$

The commutation relations between these vector fields are shown in the table 1 , and the entry in the $\mathrm{i}$-th row and $\mathrm{j}$-th column is determined as $\left[X_{i}, X_{j}\right]=$ $X_{i} X_{j}-X_{j} X_{i}, i, j=1,2,3$.

\begin{tabular}{c|ccc}
{$\left[X_{i}, X_{j}\right]$} & $X_{1}$ & $X_{2}$ & $X_{3}$ \\
\hline$X_{1}$ & 0 & $-X_{2}$ & $-5 X_{3}$ \\
$X_{2}$ & $X_{2}$ & 0 & 0 \\
$X_{3}$ & $5 X_{3}$ & 0 & 0
\end{tabular}

Table 1: Commutation relations between vector fields.

\section{Group Invariant Solutions}

For attain the generated group transformations by the $X_{i}$ for $i=1,2,3$, we should to solve the follow system of three ODEs

$$
\begin{aligned}
& \frac{d x^{*}}{d \varepsilon}=\xi_{i}^{1}\left(x^{*}(\varepsilon), t^{*}(\varepsilon), u^{*}(\varepsilon)\right), x^{*}(0)=x, \\
& \frac{d t^{*}}{d \varepsilon}=\xi_{i}^{2}\left(x^{*}(\varepsilon), t^{*}(\varepsilon), u^{*}(\varepsilon)\right), t^{*}(0)=t, \quad i=1,2,3, \\
& \frac{d u^{*}}{d \varepsilon}=\eta_{i}\left(x^{*}(\varepsilon), t^{*}(\varepsilon), u^{*}(\varepsilon)\right), u^{*}(0)=u .
\end{aligned}
$$


Exponentiation the infinitesimal symmetries of equation (1), we get the one-parameter groups $G_{i}(\varepsilon)$ generated by $X_{i}$ for $\mathrm{i}=1,2,3$.

$$
\begin{aligned}
& G_{1}(\varepsilon):(x, t, u) \rightarrow\left(x e^{\varepsilon}, t e^{5 \varepsilon}, u e^{-2 \varepsilon}\right) \\
& G_{2}(\varepsilon):(x, t, u) \rightarrow(x+\varepsilon, t, u) \\
& G_{3}(\varepsilon):(x, t, u) \rightarrow(x, t+\varepsilon, u)
\end{aligned}
$$

Theorem 3. If $u=f(x, t)$ is a solution of the Sawada-Kotera equation, so are the functions

$$
\begin{aligned}
& G_{1}(\varepsilon) f(x, t)=f\left(x e^{-\varepsilon}, t e^{-5 \varepsilon}\right) e^{-2 \varepsilon}, \\
& G_{2}(\varepsilon) f(x, t)=f(x-\varepsilon, t), \\
& G_{3}(\varepsilon) f(x, t)=f(x, t-\varepsilon) .
\end{aligned}
$$

Proof. A symmetry group of (2) is a local group of transformations $G$ with the property that for solution $u=f(x)$ of (2) and defined $g . f$ for $g \in G$, then $u=g . f(x)$ is also a solution of (2)[17].

For the one-parameter group

$$
G_{1}(\varepsilon) f(x, t)=f\left(x e^{-\varepsilon}, t e^{-5 \varepsilon}\right) e^{-2 \varepsilon} .
$$

If $f(x, t)$ is any function, then its transform by $G_{1}(\varepsilon)$ is

$$
\tilde{u}=e^{-2 \varepsilon} u=e^{-2 \varepsilon} f(x, t),
$$

which now be written in form

$$
(\tilde{x}, \tilde{t})=G_{1}(\varepsilon) \cdot(x, t)=\left(x e^{\varepsilon}, t e^{5 \varepsilon}\right),
$$

therefore

$$
\tilde{u}=e^{-2 \varepsilon} f\left(\tilde{x} e^{-\varepsilon}, \tilde{t} e^{-5 \varepsilon}\right) .
$$

The proof for $G_{2}, G_{3}$ are similar above.

\section{Optimal System of Sawada-Kotera Equation}

In this paper we want to divide the set of all invariant solutions of a given differential equation into equivalence classes. If one solution can be mapped to the other solution by a point symmetry of the PDE, then these solutions are equivalent. Classification greatly simplifies the problem of determining all invariant solutions. We need only to find one invariant solution from each class, then the whole class can be constructed by applying the symmetries. This strategy minimizes the effort needed to obtain invariant solutions[20]. 
Definition 4. The solutions $u=f(x)$ and $u=\bar{f}(x)$ are equivalent if a symmetry maps one to the other. Similarly, the symmetry maps $X$ to $\bar{X}$, so these generators are regarded as equivalent. It is important to classify invariant solutions by classifying the associated symmetry generators. Having done this, one generator from each class is used to obtain the desired set of invariant solutions. An optimal system of generators is a set consisting of exactly one generator from each class.[21].

To obtain an optimal system of subgroups, we can obtain an optimal system of subalgebras. Because these are evidently equivalent. The optimal system for one-dimensional subalgebras is the same as classification of the orbits for the adjoint representation. An adjoint representation $A d\left(\exp \left(\varepsilon X_{i}\right)\right)$ is defined by the Lie series

$$
\operatorname{Ad}\left(\exp \left(\varepsilon \cdot X_{i}\right) \cdot X_{j}\right)=X_{j}-\varepsilon\left[X_{i}, X_{j}\right]+\frac{\varepsilon^{2}}{2}\left[X_{i},\left[X_{i}, X_{j}\right]\right]-\ldots,
$$

where $\left[X_{i}, X_{j}\right]$ is the commutator for the Lie algebra, $\varepsilon$ is a parameter, and $\mathrm{i}, \mathrm{j}=1,2,3[17]$. We list all the adjoint representations of the generators of algebra Sawada-Kotera equation in Table 2, where the (i, j)-th entry indicates $A d(\exp (\varepsilon X i)) X j$.

\begin{tabular}{c|ccc}
$\operatorname{Ad}\left(\exp \left(\varepsilon \cdot X_{i}\right) \cdot X_{j}\right)$ & $X_{1}$ & $X_{2}$ & $X_{3}$ \\
\hline$X_{1}$ & $X_{1}$ & $e^{\varepsilon} X_{2}$ & $e^{5 \varepsilon} X_{3}$ \\
$X_{2}$ & $X_{1}-\varepsilon X_{2}$ & $X_{2}$ & $X_{3}$ \\
$X_{3}$ & $X_{1}-5 \varepsilon X_{3}$ & $X_{2}$ & $X_{3}$
\end{tabular}

Table 2: The adjoint representation of the Sawada-Kotera equation.

Theorem 5. An optimal system of one-dimensional Lie algebras of the Sawada-Kotera equation is provided by

$$
\text { (i) : } X_{1}, \quad(i i): X_{2}, \quad(i i i): X_{2}+X_{3}, \quad(i v): X_{2}-X_{3}
$$

Proof. Consider the symmetry algebra of the Eq.(1) whose adjoint representation was determined in table 2 and let $F_{i}^{s}(X)=A d\left(\exp \left(\varepsilon X_{i}\right) X\right)$ is a linear map, for $\mathrm{i}=1,2,3$. The matrices $M_{i}^{\varepsilon}, i=1,2,3$, with respect to basis $\left\{X_{1}, X_{2}, v_{3}\right\}$ are

$$
\begin{aligned}
M_{1}^{\varepsilon} & =\left(\begin{array}{ccc}
1 & 0 & 0 \\
0 & e^{-\varepsilon} & 0 \\
0 & 0 & e^{-5 \varepsilon}
\end{array}\right), M_{2}^{\varepsilon}=\left(\begin{array}{lll}
1 & 0 & 0 \\
\varepsilon & 1 & 0 \\
0 & 0 & 1
\end{array}\right), \\
M_{3}^{\varepsilon} & =\left(\begin{array}{ccc}
1 & 0 & 0 \\
0 & 1 & 0 \\
5 \varepsilon & 0 & 1
\end{array}\right) .
\end{aligned}
$$


Let us consider

$$
X=a_{1} X_{1}+a_{2} X_{2}+a_{3} X_{3},
$$

is a nonzero vector. Our task is to simplify it by utilizing convenient adjoint maps. Assume first that $a_{1} \neq 0$. If necessary, We can suppose $a_{1}=1$. According to table 2 , if we accomplish on such a $X$ by $A d\left(\exp \left(\frac{1}{5} a_{3} X_{3}\right)\right) X$, we can make the coefficient of $X_{3}$ vanish:

$$
X^{\prime} a=A d\left(\exp \left(\frac{1}{5} a_{3} X_{3}\right)\right) X=X_{1}+a_{2} X_{2}
$$

Then we accomplish on $X^{\prime a}$ using $A d\left(\exp \left(a_{2} X_{2}\right)\right) X^{\prime a}$ to vanish the coefficient $X_{2}$, so that $X$ is equivalent to $X^{\prime \prime} a=X_{1}$ under the adjoint representation. The remaining one-dimensional subalgebras are spanned by vectors of the above form with $a_{1}=0$. If $a_{2} \neq 0$, we put $a_{2}=1$, and then we have

$$
X^{\prime} b=X_{2}+a_{3} X_{3}
$$

We can further act on $X^{\prime} b$ by the group generated by $X_{1}$, this has the net effect of scaling the coefficient of $X_{2}, X_{3}$ :

$$
X^{\prime \prime b}=A d\left(\exp \left(\varepsilon X_{1}\right)\right) X^{\prime b}=a_{2} e^{\varepsilon} X_{2}+a_{3} e^{5 \varepsilon} X_{3} .
$$

This is a scalar multiple of $X^{\prime \prime \prime} b=X_{2}+a_{3}^{\prime} e^{4 \varepsilon} X_{3}$, so, depending on the sign of $a_{3}^{\prime}$, we can make the coefficient of $X_{3}$ either $+1,-1$ or 0 . Thus any one-dimensional subalgebra spanned by $X$ with $a_{1}=0, a_{2} \neq 0$ is equivalent to one spanned by either $X_{2}, X_{2}-X_{3}, X_{2}+X_{3}$. The further simplifications are not possible. Then an optimal system of the Sawada-Kotera equation is given by

$$
(i): X_{1}, \quad(i i): X_{2}, \quad(i i i): X_{2}+X_{3}, \quad(i v): X_{2}-X_{3} \text {. }
$$

\section{Symmetry Reduction and Differential Invariants for the Sawada-Kotera Equation}

In this section we derive the symmetries and corresponding reductions of the Sawada-Kotera equation. The Sawada-Kotera equation is expressed in terms of $(x, t, u)$, so we should to used a specific coordinates for this equation in order to reduce it. For this purpose, we should to reduce the number of independent variables of the Sawada-Kotera equation. We consider the reduction in the number of independent variables as a way to convert an original PDE into a 
simpler PDE or an ODE. we can convert the Sawada-Kotera in terms of $(x, t, u)$ into an ODE in terms of $(r, z)$. Now using the chain rule and the new obtained coordinate, we can obtain the reduced equation. For the operator,

$$
\text { (a) : } X_{1}=x \frac{\partial}{\partial x}+5 t \frac{\partial}{\partial t}-2 u \frac{\partial}{\partial u},
$$

we have

$$
\frac{d x}{x}=\frac{d t}{5 t}=\frac{d u}{-2 u}
$$

the similarity variables are as follows:

$$
r=\frac{t}{x^{5}}, \quad z=x^{2} u
$$

a solution of our equation in this case is

$$
z=f(r) \Rightarrow x^{2} u=f(r) \Rightarrow u=\frac{1}{x^{2}} f(r),
$$

we substitute it into (1) to determine the form of the function $f(r)$. We obtain that $f(r)$ has to satisfy the equation:

$$
\begin{aligned}
& -720 f(r)-180 f^{2}(r)+(1-7640 r) f^{\prime}(r)-10 f(r) f^{2}(r) \\
& -25 r f^{\prime}(r) f^{2}(r)+2750 r^{2} f^{\prime \prime}(r)-2550 r f(r) f^{\prime}(r)-1250 r^{2} f^{\prime 2}(r) \\
& -625 r^{3} f^{\prime}(r) f^{\prime \prime}(r)-172500 r^{3} f^{(3)}(r)-3000 r^{2} f(r) f^{\prime \prime}(r) \\
& -625 r^{3} f(r) f^{(3)}(r)-43750 r^{4} f^{(4)}(r)-3125 r^{5} f^{(5)}(r)=0 .
\end{aligned}
$$

Similar as above, for

$$
\text { (b) : } X_{2}=\frac{\partial}{\partial x}
$$

we obtain:

$$
\begin{aligned}
& \frac{d x}{1}=\frac{d t}{0}=\frac{d u}{0} \\
& r=t, \quad z=u, \\
& z=f(r) \Rightarrow u=f(r),
\end{aligned}
$$

when substituting it into (1), we perceive that $f(r)$ should satisfy the equation

$$
\begin{aligned}
& f^{\prime}(r)=0 . \\
& (c): X_{3}=\frac{\partial}{\partial t},
\end{aligned}
$$


The characteristic equation of this case is:

$$
\begin{aligned}
& \frac{d x}{0}=\frac{d t}{1}=\frac{d u}{0}, \\
& r=x, \quad z=u, \\
& z=f(r) \Rightarrow u=f(r),
\end{aligned}
$$

with the perceived form of $f(r)$ when substituting it into (1). After substituting we obtain that $f(r)$ has to be a solution of the following equation:

$$
\begin{gathered}
5 f^{2}(r) f^{\prime}(r)+5 f^{\prime}(r) f^{\prime \prime}(r)+5 f(r) f^{(3)}(r)+f^{(5)}(r)=0 . \\
(d): \text { For } X_{2}+X_{3}=\frac{\partial}{\partial x}+\frac{\partial}{\partial t}
\end{gathered}
$$

we have

$$
\begin{aligned}
& \frac{d x}{1}=\frac{d t}{1}=\frac{d u}{0}, \\
& r=t-x, \quad z=u, \\
& z=f(r) \Rightarrow u=f(r),
\end{aligned}
$$

when substituting it into (1), we perceive that $f(r)$ should satisfy the equation:

$$
\begin{gathered}
f^{\prime}(r)\left(1-5 f^{2}(r)-5 f^{\prime \prime}(r)\right)-5 f(r) f^{(3)}(r)-f^{(5)}(r)=0 . \\
(e): \text { Lastly, } X_{2}-X_{3}=\frac{\partial}{\partial x}-\frac{\partial}{\partial t}
\end{gathered}
$$

for this operator, the characteristic equation and independent invariants are:

$$
\begin{aligned}
& \frac{d x}{1}=\frac{d t}{-1}=\frac{d u}{0}, \\
& r=-t-x, \quad z=u, \\
& z=f(r) \Rightarrow u=f(r),
\end{aligned}
$$

the form of the function $f(r)$ is obtained with substituting it into (1), then it should satisfy the equation:

$$
-f^{\prime}(r)\left(1+5 f^{2}(r)+5 f^{\prime \prime}(r)\right)-5 f(r) f^{(3)}(r)-f^{(5)}(r)=0 .
$$




\section{Conclusion}

In this work, we used the Lie group method to find the Lie point symmetries of the Sawada-Kotera equation, which concludes to similarity variables. We utilized the corresponding invariant solutions to reduce the number of independent variables in the Sawada-Kotera equation. Also, we have obtained an optimal system for the Sawada-Kotera equation.

\section{References}

[1] E. Fan, Extended tanh-function method and its applications to nonlinear equations, Phys. Lett. A, 277 (2000), 212-218.

[2] A.M. Wazwaz, The tanh-coth method for solitons and kink solutions fornon-linear parabolic equations, Appl. Math. and Comp., 188 (2007), 1467-1475.

[3] H. Jafari, N. Kadkhoda, A. Biswas, The $\left(\frac{G^{\prime}}{G}\right)$-expansion method for solutions of evolution equations from isothermal magnetostatic atmospheres, Jour. of King Saud Uni-Sci, (2013) 25, 57-62.

[4] E. Salehpour, H. Jafari, N. Kadkhoda, Application of $\left(\frac{G^{\prime}}{G}\right)$-expansion method to nonlinear Lienard equation, Indian Journal of Sci. and Tech., 5 (2012), 2554-2556.

[5] H. Jafari, A. Borhanifar, S.A. Karimi, New solitary wave solutions for the bad Boussinesq and good Boussinesq equations, Numer. Meth. for Par. Diff. Eq., 25, No. 5 (2009), 12311237.

[6] A.M. Wazwaz, The sine-cosine method for obtaining solutions with compact and noncompact structures, Appl. Math. and Comp., 159 (2004), 559-576.

[7] H. Jafari, N. Kadkhoda, C.M. Khalique, Travelling wave solutions of nonlinear evolution equations using the simplest equation method, Comp. and Math. with Appl., 64, No. 6 (2012), 2084-2088.

[8] K. Sawada, T. Kotera, Prog. Theor. Phys., 51 (1974), 1355.

[9] B. Fuchssteiner, W. Oevel, J. Math. Phys., 23 (1982), 358.

[10] R.N. Aiyer, B. Fuchssteiner, W. Oevel, J. Phys. A, 19 (1986), 3755.

[11] D. Levi, O. Ragnisco, Inverse Problems, 4 (1988), 815.

[12] C. Liu, Z. Dai, Exact soliton solutions for the fifth-order Sawada-Kotera equation, Appl. Math. and Comp., 206 (2008), 72-275.

[13] Q. Feng, B. Zheng, Traveling Wave Solutions for the Fifth-Order Sawada-Kotera Equation and the General Gardner Equation by $\left(\frac{G^{\prime}}{G}\right)$-Expansion Method, WSEAS Tran. on Math., 9, No. 3 (2010), 171-180.

[14] A.M. Wazwaz, The extended tanh method for new solitons solutions for many forms of the fifth-order KdV equations, Appl. Math. and Comp., 184 (2007), 1002-1014.

[15] N.H. Ibragimov, Handbook of Lie Group Analysis of Differential Equations, Volumes 1, 2, 3, CRC Press, Boca Raton, Ann Arbor, London, Tokyo (1994,1995,1996). 
[16] L.V. Ovsyannikov, Group Analysis of Differential Equations, Academic Pres, New York (1982).

[17] P.J. Olver, Applications of Lie Groups to Differential Equations, Graduate Texts in Mathematics, 107, 2-nd Edition, Springer-Verlag, Berlin (1993).

[18] G. Baumann, Symmetry Analysis of Differential Equations with Mathematica, Telos, Springer Verlag, New York (2000).

[19] G.W. Bluman, S.C. Anco, Symmetry and Integtation Methods for Differential Equations, Springer-Verlag, New York (2002).

[20] M. Nadjafikhah, F. Ahangari, Symmetry Reduction of Two-Dimensional Damped Kuramoto-Sivashinsky Equation, Commun. Theor. Phys., 56 (2011), 211-217.

[21] P.E. Hydon, Symmetry Methods For Differential Equations A Beginner's Guide, Cambridge (2000).

[22] G.W. Bluman, S. Kumei, Symmetries and Differential Equations, Applied Mathematical Sciences, 81, Springer-Verlag, New York (1989).

[23] G. Gasper, M. Rahman, Basic Hypergeometric Series, Cambridge University Press, Cambridge (1990).

[24] M. Rosenblum, Generalized Hermite polynomials and the Bose-like oscillator calculus, In: Operator Theory: Advances and Applications, Birkhäuser, Basel (1994), 369-396.

[25] D.S. Moak, The q-analogue of the Laguerre polynomials, J. Math. Anal. Appl., 81 (1981), 20-47.

[26] A.G. Johnpillai, C.M. Khalique, Lie group classification and invariant solutions of mKdV equation with time-dependent coefficients, Communications in Nonlinear Science and Numerical Simulation, 16 (2011), 1207-1215. 